\title{
Performance analysis of ATM/DQDB interworking
}

\author{
Christiansen, Henning; Kvols, Kenn
}

Published in:

Proceedings of the 17th Conference on Local Computer Networks

Link to article, DOI:

10.1109/LCN.1992.228175

Publication date:

1992

Document Version

Publisher's PDF, also known as Version of record

Link back to DTU Orbit

Citation (APA):

Christiansen, H., \& Kvols, K. (1992). Performance analysis of ATM/DQDB interworking. In Proceedings of the 17th Conference on Local Computer Networks (pp. 42-49). IEEE. https://doi.org/10.1109/LCN.1992.228175

\section{General rights}

Copyright and moral rights for the publications made accessible in the public portal are retained by the authors and/or other copyright owners and it is a condition of accessing publications that users recognise and abide by the legal requirements associated with these rights.

- Users may download and print one copy of any publication from the public portal for the purpose of private study or research.

- You may not further distribute the material or use it for any profit-making activity or commercial gain

- You may freely distribute the URL identifying the publication in the public portal

If you believe that this document breaches copyright please contact us providing details, and we will remove access to the work immediately and investigate your claim. 


\section{Performance Analysis of ATM/DQDB Interworking}

\author{
Henning Christiansen \\ Technical University of Denmark \\ Building 321 \\ Denmark, DK-2800 Lyngby
}

\author{
Kenn Kvols \\ Copenhagen Telephone Company \\ Nørregade 21 \\ Denmark, DK-1199 Copenhagen K
}

\begin{abstract}
This paper considers the cell loss ratio and cell delay variation of a $D Q D B$ network, receiving traffic from a number of ATM connections. Every connection carries either connection oriented or connectionless traffic. In the analysis of the access to the bus, we realize that consecutive service times of the local access queue are correlated. We present two models, of which one is including the correlation. Finally we illustrate the correlation effect and evaluate the models by a number of simulation cases.
\end{abstract}

\section{Introduction}

In the years to come an increasing number of networks are to be inter-connected. Many Local Area Networks (LAN) already exist, Metropolitan Area Networks (MAN) will be implemented soon and ATM (Asynchronous Transfer Mode) networks are to come within a few years. Proper strategies for the interworking units (IWU's), which interconnect the networks seem rather urgent. The IWU strategies depend very much on the services the networks might offer and of the type of the interworking networks.

This paper considers ATM/DQDB interworking only, but we expect that the modelling principles presented can be generalized to other interworking examples. As the ATM cell and DQDB slot are compatible [13] the basic data unit is denoted a cell. We investigate how flows of cells, all characterized by their peak rates, are transmitted from an ATM network to a DQDB network. No further parameters are included, as the peak rate is the only parameter, for ATM connections, with a stable definition within CCITT at the moment.

From a performance analysis point of view an IWU can be regarded as a black box that performs reassembling, segmentation and re-addressing functions, furthermore it is able to buffer data. In case of $\mathrm{CO}$ traffic the IWU has to be transparent i.e. the only operation performed on an arriving ATM cell before it is transmitted to the DQDB network is a re-addressing function (VCI conversion). In case of CL traffic the IWU can be regarded as transparent also, if pipe-lining is used and no buffering of data is performed, so that cells can be transmitted to the local access queue in the DQDB network as soon as they arrive from the A'TM network. Throughout this paper transparent IWU's, both regarding CO and CL traffic, are considered only.

In an ATM based network there is no flow control or error recovery i.e. the Quality of Service (QoS) experienced at the entrance to the DQDB network should be kept within certain limits. Relevant QoS parameters are cell loss ratio in case of connection less (CL) traffic, and absolute cell delay and cell delay variation in case of connection oriented (CO) traffic. An important task is to develop methods which can predict those parameters, for example to be able to accept a new $\mathrm{CO}$ connection.

As the modelling of DQDB is very difficult due to the large number of dependent actions in the network, the number of analytical models presented in literature is very limited. Furthermore, the work has mainly been considering the unfairness of the protocol $([7],[12],[6])$ or different ways to improve the performance of the protocol $([11],[15],[14])$. In [17] and [16] close-form solutions for the medium access delay is derived using the technique of embedded Markov models. Based on certain assumptions of independence a simple expression for the delay has been obtained in [5].

The aim of this paper is to evaluate the cell loss ratio (CLR) and the cell delay variation (CDV) of a DQDB network, when CO and CL traffic flows are transmitted into the DQDB network via the same local access queue. We have adopted the idea to provide $\mathrm{CO}$ traffic by use of the queued arbitrated mode.

We have chosen to model the access to the bus by a one server queuing system, where cells from the local 
access queue are "served" by the DQDB access mechanism. The model is kept quite general in order to limit the number of detailed assumptions about the configuration of the DQDB network.

Since it turns out that two consecutive service times are positive correlated, a correlation analysis is carried out and the correlation effect is introduced in the modelling. However, for the sake of convenience a model where the correlation effect are neglected is also presented.

In the next section we describe the modelling of the access to a DQDB network, while section 3 reports some results. Finally we conclude.

\section{Modelling}

We consider a DQDB network where the $m$ 'th station is receiving data from a number of ATM connections via an interworking unit, see figure 1 . Every ATM connection carries either CO or CL traffic and is characterized by its peak rate only. As the IWU is transparent we have chosen to model the aggregated cell arrival process to the local access queue by a Poisson process with rate $\lambda$. Only the Queue Arbitrated

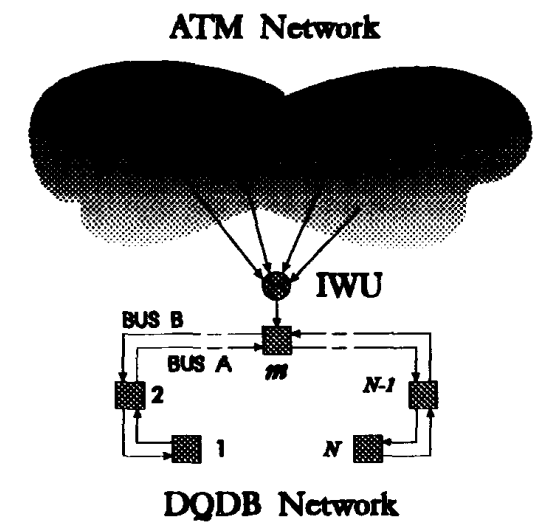

Figure 1: The considered configuration

mode with one priority level is considered. The arrival processes of requests and busy slots at station $m$ are modelled by independent "memoryless" processes (Bernoulli/Poisson). Slots on bus $A$ are busy with probability $\alpha$ and slots on Bus B carry a request with probability $\beta$. According to [5] these assumptions seem reasonable when the DQDB stations are separated by only a few slot lengths and the total load of Bus $A$ is less than about $90 \%$, and there are single arrival processes to each station. $\alpha$ and $\beta$ are estimated

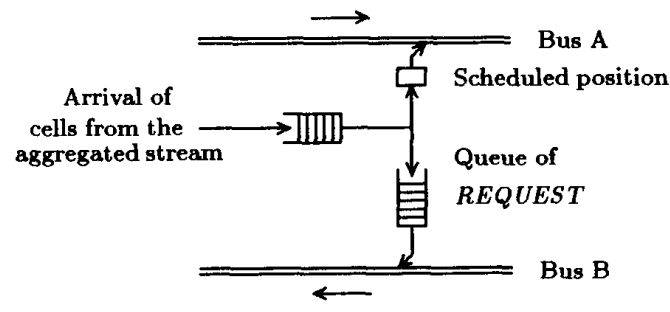

Figure 2: The access to one bus

by the average value of the offered load:

$$
\begin{aligned}
& \alpha=\sum_{i=1}^{m-1} \sum_{j=i+1}^{N} \lambda_{i j} \tau \\
& \beta=\sum_{i=m+1}^{N} \sum_{j=i+1}^{N} \lambda_{i j} \tau
\end{aligned}
$$

where $\lambda_{i j}$ is the traffic intensity from station $i$ to $j$ and $\tau$ is the duration of a slot.

The modelling approach presented here is especially adequate if the stations do not cause heavy load situations to occur.

As details about the DQDB medium access protocol can be found in [1], we will only mention those characteristics being relevant for our modelling purposes. The station can have at most one cell in the "scheduled position" waiting for transmission on bus A, see figure 2 . The waiting time $(W)$ in this position is controlled by the request- and countdown counters, and it can be obtained as the sojourn time in the virtual queueing system illustrated in figure 3 . The arrival process is the aggregated stream of requests from stations with a number higher than $m$ and cells from the local access queue, both having a service requirement of one empty slot. The service time is the waiting time until the next empty slot on Bus $\mathrm{A}$ arrives (geometric distributed with mean $1 /(1-\alpha)$ ).

When the waiting time for a cell in the scheduled position is applied as the service time for cells in the local access queue, the cell delay variation and cell loss ratio can be evaluated. However, two problems arise:

(a) It is not possible to derive a close form expression for the waiting time $W$.

(b) The service times are correlated.

Problem (a) arises because only one cell at a time is allowed entrance to the virtual queueing system. An 


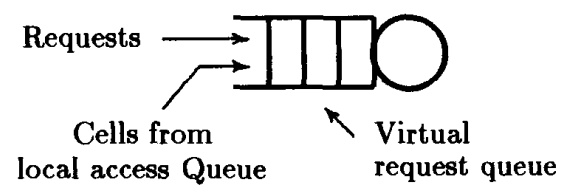

Figure 3: The service time is equal to the sojourn time $W$ in a virtual queueing system where requests and cells are served by empty slots.

exact expression cannot be derived, but "best" and worst cases can be found. A "best" case is obtained if we ignore the cell arrivals to the virtual queue. This results in a simple discrete time Markov chain. In appendix A the sojourn time is found to be geometrically distributed:

$$
\begin{aligned}
\operatorname{Prob}\{W=n \tau\} & =p_{b}\left(1-p_{b}\right)^{n-1}, n=1,2, \ldots \\
p_{b} & =\frac{1-\alpha-\beta}{1-\beta}
\end{aligned}
$$

Alternatively; if we assume that the aggregated stream is a Bernoulli process with rate $\beta+\lambda \tau$, we obtain a worst case expression for $W$, i.e. geometrically distributed but with parameter $p_{w}$ :

$$
\begin{aligned}
\operatorname{Prob}\{W=n \tau\} & =p_{w}\left(1-p_{w}\right)^{n-1}, n=1,2, . \\
p_{w} & =\frac{1-\alpha-(\beta+\lambda \tau)}{1-(\beta+\lambda \tau)}
\end{aligned}
$$

The distribution of the real waiting time is close to the worst case distribution if $\lambda \tau \ll \beta$. However, if $\lambda \tau>\beta$ the aggregated arrival process to the virtual queueing system is dominated by cell arrivals from the local access queue, and equation 6 is a very "pessimistic" estimate. To overcome this problem we have in appendix $\mathrm{B}$ derived an alternative worst case distribution for the waiting time by transferring the Bernoulli processes into similar Poisson processes. The waiting time $W$ is found to be exponentially distributed:

$$
\begin{aligned}
& W \in \operatorname{Exp}\left(\mu_{w}\right) \\
& \mu_{w}=\frac{1-\alpha-\beta}{\tau}
\end{aligned}
$$

When $\lambda \tau \gg \beta$ the waiting time distribution given by (7) will be less pessimistic than the distribution given by (5) because (7) takes into account that only one cell at a time is allowed entrance to the virtual queue.

Problem (b) stated above arises because a long service time for a cell implies that a large number of requests are received during the service time of this cell.
Since the service time of the consecutive cell is mainly equal to the time it takes to "serve" these requests, the service time of the consecutive cell is positively correlated with the preceding cell. In appendix $\mathrm{C}$ the correlation between the service time of two cells (no. $n$ and $n+m$ ) within a busy period (see figure 7 ) is found:

$$
\begin{aligned}
\operatorname{Cor}\left\{T_{n}, T_{n+m}\right\} & =[\gamma(n)]^{m} \\
\lim _{n \rightarrow \infty} \gamma(n)=\gamma & =\frac{\beta}{1-\alpha}
\end{aligned}
$$

In this paper we define the contribution to cell delay variation (CDV) caused by the DQDB network as the difference in sojourn time experienced by two consecutive cells. The sojourn time is defined as the time in the local access queue plus the service time and is denoted $U$. In order to get a conservative estimate, the sojourn time of the first cell is set to zero implying that the CDV is equal to the maximum sojourn time. This approach yields reasonable results if the aggregated cell arrival process consists of a large number of cell streams, so that consecutive cells belonging to a particular cell stream are separated by multiple cell arrivals from other streams. In the next section we evaluate CDV by the fraction of cells gaining a higher sojourn time than a certain threshold value.

The cell loss ratio is evaluated similar to the CDV; we are regarding the fraction of cells which arrives to the queue at an instant where the number of buffered cells (including the cell under service) exceed a certain threshold $(\kappa)$. Though it is not possible to truncate (because of the correlation effect) to obtain the loss ratio at a certain buffer size, we choose to evaluate the CLR in this way due to the higher information.

In the following we present two models of the configuration illustrated in figure 1 . The first neglects the correlation effect resulting in a simple model. The second incorporates the correlation effect which yields a more accurate but complex model.

\section{Model I}

Model I assumes that consecutive service times are independent and all exponentially distributed. This results in a $M / M / 1$ model, where the arrival rate is $\lambda$ and the service rate is $\mu$, where the service rate is found as the maximum of:

$$
\mu=\max \left(\frac{p_{w}}{\tau}, \mu_{w}\right)
$$


$p_{w}$ and $\mu_{w}$ follows from equation 6 and 8. The probability of at least $\kappa$ cells in the system is:

$$
\operatorname{Prob}\{\text { at least } \kappa \text { cells }\}=\left(\frac{\lambda}{\mu}\right)^{\kappa}
$$

and the distribution of the sojourn time $U$ is exponential:

$$
U \in \operatorname{Exp}(\mu-\lambda)
$$

\section{Model II}

The correlation effect is included in model II by modelling the server with a two state Markov modulated service rate, see figure 4 .

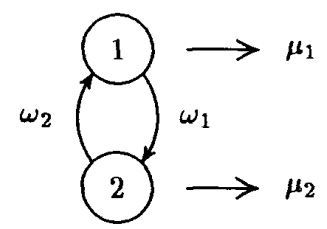

Figure 4: Model of the server

The worst case is obtained when $\mu_{1}$ is equal to zero. This implies that the service of a cell always ends when the server is at state 2 and that the service of a consecutive cell will start at state 2. Assuming that all service times start with the server at state 2 , the service times become equal and independent hyper-exponentially distributed. The resulting queueing system, which is a special case of a $\mathrm{M} / \mathrm{PH} / 1$ queue, is dealt with in chapter 3 in [2].

$\omega_{1}$ and $\omega_{2}$ are determined in the following way: In a time interval $t$ there will, on average, be served $\mu t$ cells (assuming that the system is within a long busy period, the service rate $\mu$ is given by eq. 11). The correlation between the first and last cell that has been served is $\gamma^{\mu t} . \exp \left(-\omega_{i} t\right), i=1,2$ is the probability of still being in state $i$ given that the server was at state $i$ at $t=0 . \exp \left(-\omega_{i} t\right), i=1,2$ is therefore a measure of the correlation between the service times of consecutive cells. If we let:

$$
\exp \left(-\omega_{1} t\right)=\exp \left(-\omega_{2} t\right)=k \gamma^{\mu t}
$$

we obtain

$$
\begin{aligned}
\omega_{1}=\omega_{2} & =-\mu \ln (\gamma)+\frac{\ln (k)}{t} \\
& =-\mu \ln (\gamma) \quad t \rightarrow \infty
\end{aligned}
$$

$\mu_{2}$ is chosen such that the average service time is equal to $1 / \mu$ where $\mu$ is given by equation 11 .

$$
\begin{aligned}
& \mu_{1}=0 \\
& \mu_{2}=2 \mu
\end{aligned}
$$

The steady state probabilities and the distribution of the sojourn time $(U)$ can be obtained from the procedure described in [2].

\section{Results}

The influence of the described characteristics of the service time is illustrated with three cases and compared with the models. The worst case concerning cell loss ratio and cell delay variation is obtained when bus $A$ is loaded as much as possible. On the other hand, in order to justify the assumptions of Bernoulli arrival processes of requests and busy slots, the total load of the bus is limited to $90 \%$. Each case considers a $150 \mathrm{Mbps}$ DQDB network, where the total load of the buses is $90 \%$. The offered load to the tagged station is $15 \mathrm{Mbps}$ ( $10 \%$ of the bus capacity) while the load from the remaining stations is $120 \mathrm{Mbps}$. In case 1 the tagged station is placed close to the front end of the bus and the stations placed before are loading the bus with a total of 7.5 Mbps, the stations placed after load totally $112.5 \mathrm{Mbps}$. In case 2 the tagged station is placed at the middle of the bus and in case 3 close to the end of the bus. The parameters are shown in table 1 .

\begin{tabular}{|c|ccc|c|}
\hline Case & 1 & 2 & 3 & Eq. \\
\hline$\alpha$ & 0.05 & 0.40 & 0.75 & $(1)$ \\
$\beta$ & 0.75 & 0.40 & 0.05 & $(2)$ \\
$\lambda \tau$ & 0.10 & 0.10 & 0.10 & - \\
\hline$\gamma$ & 0.79 & 0.67 & 0.20 & $(10)$ \\
$\gamma$ & 0.80 & 0.33 & 0.21 & $(4)$ \\
$p_{b}$ & 0.67 & 0.20 & 0.12 & $(6)$ \\
$p_{w}$ & 0.20 & 0.20 & 0.20 & $(8)$ \\
$\mu_{w} \tau$ & 0.67 & 0.20 & 0.20 & $(11)$ \\
$\mu \tau$ & 0.67 \\
$\frac{\lambda}{\mu}$ & 0.15 & 0.50 & 0.50 & - \\
\hline
\end{tabular}

Table 1: Configurations in the cases. "Eq." denotes the number of the equation, where the variable is obtained.

The complementary distribution of the number of cells in the system $(\kappa)$ seen by an arriving cell is shown 

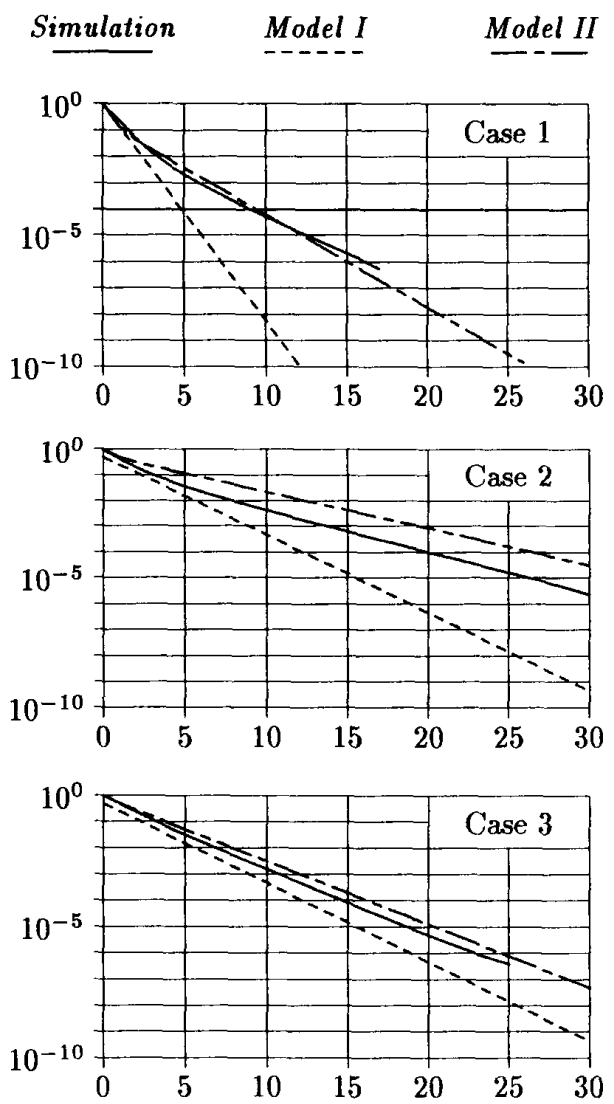

Figure 5: The probability of an arriving cell experience at least $\kappa$ cells in the system vs. $\kappa$.

in figure 5. Two observations can be made from the simulations ${ }^{1}$ :

First, the initial slope ( $\kappa$ small) is less steep in case 2 and 3 than in case 1 , because the service rate is significantly higher in case 1 . Second, in case 2 and especially in case 1 there is a bend in the slope due to the correlation effect. Long service times are more likely to be followed by long service times, which implies that the local access queue will build up to a higher level compared to a system with no correlation. From table 1 we see that the correlation is highest in case 1 resulting in the biggest change in slope.

Model I seems to estimate the system reasonably for small $\kappa$ values, but is unable to model the correlation effect. In case 1 the service rate is obtained from eq. 6 ,

\footnotetext{
${ }^{1}$ In order to be able to simulate a larger number of cell arrivals, the simulations have been carried out at one station only, based on the assumption of Bernoulli arrival processes of request and empty slots.
}
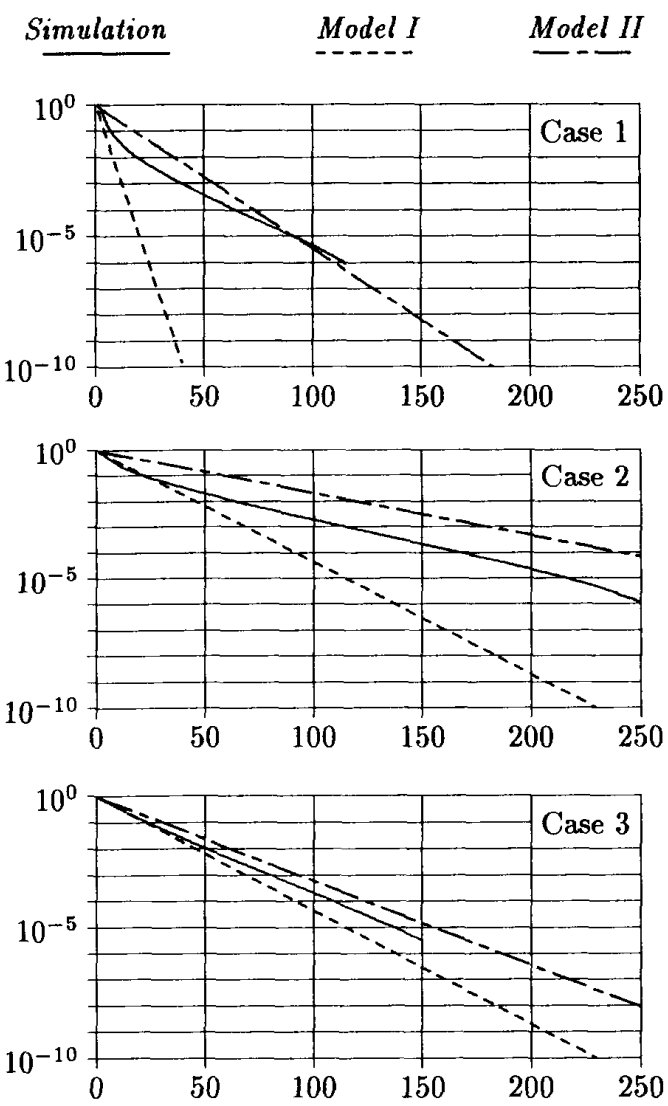

Figure 6: The complementary distribution of the waiting time (time slots) of a cell in the system.

while the service rate in case 3 is chosen from eq. 8 (in case 2 both formulas result in the same service rate). Model II is able to estimate the correlation reasonably, though it is underestimating the slope in case 1.

In general it requires short buffers only (less than $100)$ in order to achieve small cell losses. In the three cases presented here this is mainly explained by a low load of the local access queue (i.e. $\lambda / \mu$ is small). However, small cell losses will also be obtained when the load increases for a given utilization of the bus $(\alpha+\beta+\lambda \tau$ fixed $)$. An increased load can only be achieved if $\lambda$ is increased and $\alpha, \beta$ correspondingly is decreased, implying that the service times are becoming more deterministic in nature. In the limit, where the load of the access queue equals the utilization of the bus, the service time will be completely deterministic and equal to one time slot. To summarize - we consider cell loss as no problem if the load of the local access queue is not unreasonably high. 
In figure 6 the distributions of the sojourn time are illustrated. The "picture" is very much alike figure 5 - the effect of the correlation and the different service rates between the cases are similar to figure 5 . In case 1 a large fraction of cells almost get no delay while about 1 of $10^{5}$ cells are delayed at least 90 time slots $(\sim 250 \mu \mathrm{sec})$. The corresponding delays for case 2 and 3 are 220 time slots $(\sim 600 \mu \mathrm{sec})$ and 140 time slots $(\sim 400 \mu \mathrm{sec})$.

\section{Conclusion}

In this paper the ATM/DQDB interworking has been investigated by analyzing the access to a DQDB network of an aggregated cell arrival process coming from an ATM network. The target for the analyze has been to evaluate the cell loss ratio (CLR) and cell delay variation (CDV). We have modelled the access to the network as a one server queue and found a worst case distribution of the service time. Futhermore, we have included the correlation between consecutive service times in the modelling.

Two simple models for the queueing behavior have been presented; one model which does not include the correlation effect and another which does. The model which includes the correlation effect seems to estimate the CLR and CDV reasonably.

The results illustrate that the cell loss ratio in general is a minor problem, while the cell delay variation can be significant, especially due to the correlation effect.

We envision the following directions for future research: If the DQDB network is applied in a multi service traffic environment where stations may to transmit large packets, the arrival processes of requests and busy slots should be modified in accordance with this. Furthermore, the effect of using the priority mechanism of DQDB for different kinds of traffic classes is a topic of importance.

\section{Acknowledgement}

The authors gratefully acknowledge support from the CEC RACE programme, project R2032 COMBINE and the Tecnical University of Denmark. Furthermore they would like to thank Prof. Peter Martini, University of Paderborn for his valueable comments.

\section{A Sojourn time in the virtual queue (discrete time model)}

The sojourn time $W$ in the queueing system illustrated in figure 3 is derived in this appendix. We assume that there are no cell arrivals (i.e. $\lambda=0$ ) and requests arrive on Bus B according to a Bernoulli process with rate $\beta$ and busy slots on Bus A with rate $\alpha$. The sequence $\left\{H_{n} \mid n=1,2, \ldots\right\}$ denotes the number of unserved requests at the tagged station immediately after an arrival of a slot on bus A (more precisely; the arrival of a busy bit on a slot). $H_{n}$ forms a Markov chain with state spaces $N_{0}$ and jump probabilities:

$$
\left\{\begin{array}{lll}
k \rightarrow k+1 & : & \alpha \beta \\
k \rightarrow k & : & \alpha(1-\beta)+(1-\alpha) \beta \\
& & =\alpha+\beta-2 \alpha \beta \\
k \rightarrow k-1 & : & (1-\alpha)(1-\beta)
\end{array}\right.
$$

If $1>\alpha+\beta$ the chain is stable and the steady state probabilities are:

$$
\begin{gathered}
\pi_{k}=\pi_{0}\left(\frac{\alpha \beta}{(1-\alpha)(1-\beta)}\right)^{k}, \quad k=0,1, \ldots \\
\pi_{0}=\frac{1-\alpha-\beta}{1-\alpha-\beta+\alpha \beta}
\end{gathered}
$$

We assume a request arrives to the tagged station and the number of requests in queueing system immediately before the arrival is $k$. The probability density function (pdf.) of the sojourn time follows a negative binomial distribution:

$$
\begin{gathered}
f(n \mid k)=\left(\begin{array}{c}
n-1 \\
k
\end{array}\right)(1-\alpha)^{k+1} \alpha^{n-1-k} \\
, n=k+1, \ldots
\end{gathered}
$$

The unconditionally pdf. is:

$$
\begin{aligned}
f(n) & =\sum_{k=0}^{n-1} \phi_{k} f(n \mid k), \quad n=1,2, \ldots \\
& =\left(1-\frac{\alpha}{1-\beta}\right)\left(\frac{\alpha}{1-\beta}\right)^{n-1}
\end{aligned}
$$

\section{B Distribution of the service time (continuous time model)}

In this appendix we derive the distribution of the service time (i.e. the time a segment spends in the 
scheduled position). We assume that the arrival processes of requests and busy slots are independent "memoryless" processes. Since we can achieve closed form solutions with a continuous time scale only, we transfer the calculations from the discrete time domain to the similar continuous time (i.e. Bernoulli processes to Poisson processes). This transfer results in a worst case consideration, due to the fact that Poisson processes are less "smooth" than the corresponding Bernoulli processes. Requests arrive with rate $\beta / \tau$ and empty slots arrive with rate $(1-\alpha) / \tau$ (eq. 1 and eq. 2). $\tau$ denotes the time of a slot.

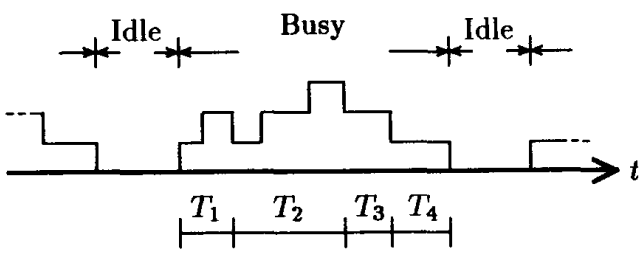

Figure 7: The number of cells in the tagged system (number of cells in local queue plus cell in scheduled position) vs. time. $T_{i}$ denotes the service time of the $i$ 'th cell in a consecutive stream of cell (busy period).

We assume that the system has been idle in a long period. When the first cell arrives, the system remains busy for a number of consecutive cell arrivals. See figure 7 .

The service time of cell $1\left(T_{1}\right)$ is obtained in a similar manner as in appendix $A$ : In an idle period the number of outstanding requests form a Markov process with state space $N_{0}$. The intensities are given by:

$$
\left\{\begin{array}{lll}
k \rightarrow k+1 & : & \beta / \tau \\
k \rightarrow k-1 & : & (1-\alpha) / \tau
\end{array}\right.
$$

This corresponds to a $M / M / 1$ queue. The distribution of the sojourn time is known to be exponentially distributed:

$$
T_{1} \in E\left(\mu_{1}\right), \quad \mu_{1}=\frac{1-\alpha-\beta}{\tau}
$$

The number of requests $\left(R_{1}\right)$ received in $T_{1}=t_{1}$ is distributed according to a Poisson distribution:

$$
R_{1} \in \operatorname{Pois}\left(\beta t_{1} / \tau\right)
$$

The unconditionally probability of $R_{1}$ is from the theory of stochastic processes known to be geometrically distributed:

$$
\operatorname{Prob}\left\{R_{1}=x\right\}=\frac{\mu_{1}}{\mu_{1}+\beta / \tau}\left(\frac{\beta / \tau}{\mu_{1}+\beta / \tau}\right)^{x}
$$

If $R_{1}=x$ the distribution of the second service time is $E_{-(x+1)}((1-\alpha) / \tau)$, i.e.:

$$
f_{x+1}(t)=\frac{[(1-\alpha) t / \tau]^{x}}{x !} \frac{1-\alpha}{\tau} e^{-(1-\alpha) t / \tau}
$$

The unconditionally distribution becomes

$$
\begin{aligned}
f(t) & =\sum_{x=0}^{\infty} \operatorname{Prob}\left\{R_{1}=x\right\} f_{x+1}(t) \\
& =\frac{\mu_{1}(1-\alpha) / \tau}{\mu_{1}+\beta / \tau} \exp \left[-\left(\frac{\mu_{1}(1-\alpha) / \tau}{\mu_{1}+\beta / \tau}\right) t\right]
\end{aligned}
$$

I.e. $T_{2}$ is exponentially distributed with rate:

$$
\mu_{2}=\frac{\mu_{1}(1-\alpha) / \tau}{\mu_{1}+\beta / \tau}=\frac{1-\alpha-\beta}{\tau}=\mu_{1}
$$

In general is

$$
T_{n} \in \operatorname{Exp}\left(\frac{1-\alpha-\beta}{\tau}\right)
$$

By transferring Bernoulli processes into similar Poisson processes we have shown that with correct modelling of the cell stream into the virtual queueing system in figure 3 , the service time for any cell is distributed according to eq. 28 .

\section{Correlation between consecutive service times}

Given that the service time of the $n$ 'th cell in a busy period (see figure 7) was $T_{n}=t_{n}$ (seconds), the number of requests received in $T_{n}$ is distributed according to a $\operatorname{Bin}\left(\beta t_{n} / \tau\right)$. The mean value becomes:

$$
\begin{aligned}
E\left\{T_{n+1} \mid T_{n}\right\} & =\sum_{i=0}^{\min \left(1, \frac{t_{n}}{\tau}\right)}\left(\begin{array}{c}
n \\
i
\end{array}\right) \beta^{i}(1-\beta)^{n-i} \frac{i+1}{1-\alpha} \\
& =\frac{n \beta+1}{1-\alpha}
\end{aligned}
$$

The covarians between $T_{n}$ and $T_{n+1}$ is:

$$
\begin{aligned}
\operatorname{Cov}\{ & \left.T_{n+1}, T_{n}\right\} \\
= & E\left\{\operatorname{Cov}\left\{T_{n+1}, T_{n} \mid T_{n}\right\}\right\} \\
& \quad+\operatorname{Cov}\left\{E\left\{T_{n+1} \mid T_{n}\right\}, E\left\{T_{n} \mid T_{n}\right\}\right\} \\
= & \frac{\beta}{1-\alpha} \operatorname{Var}\left\{T_{n}\right\}
\end{aligned}
$$


The correlation then becomes:

$$
\begin{aligned}
\gamma(n) & =\frac{\operatorname{Cov}\left\{T_{n+1}, T_{n}\right\}}{\sqrt{\operatorname{Var}\left\{T_{n+1}\right\} \operatorname{Var}\left\{T_{n}\right\}}} \\
& =\frac{\beta}{1-\alpha} \sqrt{\frac{\operatorname{Var}\left\{T_{n}\right\}}{\operatorname{Var}\left\{T_{n+1}\right\}}}
\end{aligned}
$$

If $n \rightarrow \infty$ then:

$$
\gamma=\frac{\beta}{1-\alpha}
$$

From eq. 30 and 32 we obtain that the correlation of $T_{n}$ and $T_{n+m}$ within a busy period, is:

$$
\operatorname{Cov}\left\{T_{n}, T_{n+m}\right\}=[\gamma(n)]^{m}
$$

\section{References}

[1] Proposed Standard: Distributed Queue Dual Bus (DQDB), Subnetwork of a Metropolitan Area Network (MAN), P.802.6/D15, IEEE, October 1, 1990.

[2] Marcel F. Neuts: Matrix-Geometric Solutions in Stochastic Models - an algorithmic approach, The John Hopkins University Press, Baltimore, Maryland $21218,1981$.

[3] Chatchik C. Bisdikian: Waiting Time Analysis in a Single Buffer DQDB (802.6) Network, IEEE Journal on selected area in communications, vol. 8 , No. 8 , October 1990, pp. 1565-1573.

[4] Chatchik C. Bisdikian: A Performance Analysis of the IEEE 802.6 (DQDB) subnetwork with the bandwidth balancing mechanism, Computer Networks and ISDN Systems 24 (1992), North-Holland, pp. 367-385.

[5] Henning Christiansen: Performance Analysis and Modelling of the Distributed Queue Dual Bus $(D Q D B)$, presented at COST 224 final seminar, Paris, October 1991.

[6] Marco Conti, Enrico Gregori, Luciano Lenzini: $A$ Methodological Approach to an Extensive Analysis of $D Q D B$ Performance and Fairness, IEEE Journal on selected area in communications, vol. 9, No. 1, January 1991.
[7] Peter Davids, Peter Martini: Performance Analysis of $D Q D B$, Proc. of the International Conference on Computers and Communications, IEEE, March 21-23 1990, pp. 548-555.

[8] Peter Davids, Peter Martini: High Speed LANs and MANs-Protocol and Problems, Information network and data communication 3: Proceedings of the IFIP TC6 3. Lillehammer, Norway, 26-29 March, 1990.

[9] Mark W. Garrett, San-Qi Li: A Study of Slot Reuse in a Dual Bus Multiple Access Networks, IEEE Journal on selected area in communications, vol. 9, No. 2, February 1991, pp. 248.

[10] Ellen L. Hahne, Abhijit K. Choudhury, Nicholas F. Maxemchuk: Improving the Fairness of the Distributed-Queue-Dual-Bus Networks, INFOCOM '90, San Francisco, June 5-7, 1990, pp. 175-184.

[11] Ahmed E. Kamal: Efficient Multi-Segment Message Transmission with Slot Reuse on DQDB, INFOCOM '91, April 7-11, 1991, pp. 869.

[12] Harbans Kaur, Graham Campbell: $D Q D B$ - an Access Delay Analysis, INFOCOM '90, San Francisco, June 5-7, 1990, pp. 630.

[13] T.D. Poelhekken, F. van den Dool, A. J. J. Kerkhof: DQDB-ATM Interworking Aspects, Conference on Local Communication Systems: LAN and PBX, June 1991.

[14] Philip G. Potter, Moshe Zukerman: Analysis of a DQDB Subnetwork with Eraser Nodes, ITC 13, Copenhagen 1991.

[15] Manoel A. Rodrigues: Erasure Node: Performance Improvements for the IEEE 802.6 MAN, INFOCOM '90, San Francisco, June 5-7, 1990, pp. 636.

[16] T. Stock, P. Tran-Gia: A Discrete Time Analysis of the DQDB Access Protocol with General Input Traffic, Contribution to the SBT/IEEE International Telecommunications Symposium, Rio de Janerio, Brazil, September 3-6, 1990.

[17] P. Tran-Gia, T. Stock: Approximate Performance Analysis of the DQDB Access Protocol, ITC Specialist Seminar, Paper No. 16.1, Adelaide, Australia, 1989. 